Check for updates

Cite this: Chem. Sci., 2019, 10, 4097

๑ All publication charges for this article have been paid for by the Royal Society of Chemistry

Received 5th February 2019

Accepted 6th March 2019

DOI: $10.1039 / c 9 s c 00644 c$

rsc.li/chemical-science

\section{Experimental and theoretical investigation of overall energy deposition in surface-induced unfolding of protein ions $\dagger$}

\author{
Micah T. Donor, ${ }^{a}$ Austin M. Mroz ${ }^{a}$ and James S. Prell (D) *ab
}

Recent advances in native mass spectrometry have enabled its use to probe the structure of and interactions within biomolecular complexes. Surface-induced dissociation, in which inter- and intramolecular interactions are disrupted following an energetic ion-surface collision, is a method that can directly interrogate the topology of protein complexes. However, a quantitative relationship between the ion kinetic energy at the moment of surface collision and the internal energy deposited into the ion has not yet been established for proteins. The factors affecting energy deposition in surface-induced unfolding (SIU) of protein monomers were investigated and a calibration relating laboratory-frame kinetic energy to internal energy developed. Protein monomers were unfolded by SIU and by collision-induced unfolding (CIU). CIU and SIU cause proteins to undergo the same unfolding transitions at different values of laboratory-frame kinetic energy. There is a strong correlation between the SIU and CIU energies, demonstrating that SIU, like CIU, can largely be understood as a thermal process. The change in internal energy in CIU was modeled using a Monte Carlo approach and theory. Computed values of the overall efficiency were found to be approximately $25 \%$ and used to rescale the CIU energy axis and relate nominal SIU energies to internal energy. The energy deposition efficiency in SIU increases with mass and kinetic energy from a low of $\sim 20 \%$ to a high of $\sim 68 \%$, indicating that the effective mass of the surface increases along with the mass of the ion. The effect of ion structure on energy deposition was probed using multiple stages of ion activation. Energy deposition in SIU strongly depends on structure, decreasing as the protein is elongated, due to decreased effective protein-surface collisional cross section and increased transfer to rotational modes.

\section{Introduction}

The ability of electrospray ionization (ESI) to preserve nativelike non-covalent interactions into the gas phase has led to the study of a range of important biological systems using native mass spectrometry. Native ion mobility-mass spectrometry (IM-MS), which can be used to characterize the overall shape and size of an ion by determining its collisional cross section (CCS) in a buffer gas, is a powerful tool for the investigation of high-order structure. ${ }^{1}$ Various gas-phase dissociation methods can be used to disrupt covalent and/or non-covalent interactions in order to probe structural characteristics such as sequence, ${ }^{2-4}$ location of ligand binding or post-translational modifications, ${ }^{3-5}$ connectivity of subunits in a complex, ${ }^{6-8}$ and

${ }^{a}$ Department of Chemistry and Biochemistry, 1253 University of Oregon, Eugene, OR 97403-1253, USA

${ }^{b}$ Materials Science Institute, University of Oregon, 1252 University of Oregon, Eugene, OR 97403-1252, USA. E-mail: jprell@uoregon.edu; Fax: +1 541346 4643; Tel: +1 541 3462597

$\dagger$ Electronic supplementary information (ESI) available. See DOI: $10.1039 / \mathrm{c} 9 \mathrm{sc} 00644 \mathrm{c}$ differences in stability between closely-related structures., ${ }^{\mathbf{9}, 10}$ These dissociation methods vary as to how the ion is activated, i.e., via charge transfer/recombination, ${ }^{11-14}$ collisions with neutral species, ${ }^{15}$ or absorption of photons. ${ }^{16-18}$

Collision induced dissociation (CID), in which ions are slowly heated by many low energy collisions with a neutral gas (e.g. Ar), is the most commonly-used method for interrogating protein complex composition using mass spectrometry. The products of CID for a non-covalently bound assembly such as a protein oligomer with $n$ subunits are typically an ejected monomer and remaining $(n-1)$-mer. ${ }^{9,19}$ For protein assembly ions, the charge of the complex is typically partitioned asymmetrically between the products - the monomer is often unfolded and carries a share of the charge that is larger than its share of the mass of the complex.,920,21 This provides useful information about the identity of the subunits in a complex but imparts only limited information about the quaternary structure of the complex.

By contrast, surface induced dissociation (SID) is an alternative dissociation method that can yield more information about the high-order structure of protein complexes than is typically obtained in CID..$^{22,23}$ In SID, dissociation is caused by 
a single high-energy collision event with a rigid surface, usually a gold electrode coated with a self-assembled monolayer of perfluorinated alkanethiol (FSAM). Unlike CID, SID often fragments native-like protein complexes such that the products remain compact on the millisecond timescale of the experiment and the charge is partitioned symmetrically. ${ }^{22,24,25}$ Seminal results from the Wysocki group for a variety of protein homoand heterooligomers indicate that the SID products in many cases reflect the quaternary structure of the complex observed in condensed-phase data (e.g. a hexamer with an X-ray crystal structure indicative of a dimer of trimers will dissociate into two trimers upon SID), and thus that SID can probe the high-order structure of protein complexes. ${ }^{\mathbf{6 - 8 , 2 6 , 2 7}}$ For protein complexes, correlation between the surface area of the interfaces broken and the SID collision energy required to break them has been reported, ${ }^{26}$ but the challenge of translating the nominal laboratory frame energies to internal energies has limited quantitative determination of protein-protein interfacial areas directly from SID data. SID has been implemented on a number of different mass spectrometry platforms, including FT-ICR ${ }^{28,29}$ and $\mathrm{Q}-\mathrm{TOF}^{30}$ instruments. In partnership with Waters Corp., the Wysocki group has developed a kit that enables routine application of SID on Waters Synapt Q-IM-TOF instruments, making this platform a testbed for SID of native protein complexes.

The energetics of SID have been previously characterized, both experimentally ${ }^{31-33}$ and computationally, ${ }^{34,35}$ for small monomeric molecules and peptides. In general, the kinetics of product formation from these precursor ions after relatively low-energy surface collision can be well-described using unimolecular RRKM theory, indicating that the dissociation process occurs away from the surface following picosecond-timescale $^{34,36}$ thermalization of the energy deposited by the collision. Multiple studies have shown that approximately $20 \%$ of the ion's laboratory frame kinetic energy is converted to internal energy of the ion for collisions with the typical FSAM surfaces. ${ }^{31,32,34,35}$ This fraction varies little with the angle of incidence, which instead modulates the proportion of the laboratory frame kinetic energy transferred out of the ion's translational modes or into the surface, with shallower angles decreasing transfer into the surface and increasing the amount of translational energy remaining after surface collision. ${ }^{31}$ For some peptide ions, fragments that appear at very short reaction times suggest that "shattering" is an alternate dissociation pathway at high incident kinetic energies (such that greater than $10 \mathrm{eV}$ is transferred to internal modes), ${ }^{33,37}$ a result supported by simulations of glycine ${ }^{38}$ and octaglycine ${ }^{39}$ SID showing that specific collision orientations lead to shattering. Shattering, in which fragmentation is nearly instantaneous following surface impact (ps timescale), is also commonly observed for small molecules and large non-covalent clusters of atoms and small molecules (e.g. Ar, ammonium) colliding with the surface at high (transonic) velocities. ${ }^{\mathbf{4 0 - 4 3}}$

Due to the vast number of degrees of freedom present in proteins and protein complexes, energy deposition and dissociation in SID can be much more difficult to study quantitatively using the same methods employed for peptides. Proteins and protein complexes also differ from peptides in that the observed fragments result from the disruption of many non-covalent interactions that can be distributed across a large surface area, rather than a single covalent bond. This raises the possibility that, when a small portion of a large species traveling at high velocity collides with the surface, shear forces and other mechanical effects may play a large role, leading to dissociation of some of these non-covalent interactions before thermalization is complete. Multiple collisions of the ion with the surface may also occur, further complicating the study of SID dynamics both computationally and experimentally. ${ }^{35}$

A first step towards understanding SID of protein complexes more quantitatively is to understand how surface collisions deposit energy into proteins more generally. Here, unfolding of protein monomers, as measured by ion mobility after surface collision, is used to investigate energy deposition upon surface collision. We demonstrate that proteins subjected to collisionand surface-induced unfolding (CIU and SIU, respectively) undergo the same unfolding transitions and quantify the relationship between their nominal laboratory-frame energy scales. Monte Carlo simulations and theoretical modeling of CIU enable calibration of the internal energy deposited for CIU, which in turn is used to calibrate overall energy deposition in SIU. We show that the efficiency of SIU, defined as the proportion of laboratory-frame kinetic energy converted to internal energy of the ion, can be nearly three times the efficiency of CIU for large monomeric proteins. Finally, SIU is shown to depend strongly on the structure of the precursor ion, with more unfolded ions having a smaller effective protein-surface CCS and lower energy transfer efficiency.

\section{Methods}

\section{Sample preparation}

Myoglobin, $\beta$-lactoglobulin, concanavalin A, carbonic anhydrase, alcohol dehydrogenase, albumin, bovine serum albumin, and transferrin were acquired from Sigma-Aldrich. Two protein components of anthrax lethal toxin, protective antigen, $\mathrm{PA}_{63}$, and the N-terminal domain of lethal factor, $\mathrm{LF}_{\mathrm{N}}$, were graciously provided by Dr Bryan Krantz at the University of Maryland. Lyophilized proteins were reconstituted in ultrapure (18 $\mathrm{M} \Omega$ ) water. Protein samples were exchanged into either $200 \mathrm{mM}$ ammonium acetate $10 \mathrm{mM}$ ammonium bicarbonate $\mathrm{pH} 8\left(\mathrm{PA}_{63}\right)$ or $200 \mathrm{mM}$ ammonium acetate $\mathrm{pH} 7$ (all other proteins) using Micro Bio-Spin 6 desalting columns (BioRad).

\section{Ion mobility-mass spectrometry}

All ion mobility-mass spectra were collected using a Synapt G2-Si ion mobility-mass spectrometer (Waters Corp.) equipped with a nanoelectrospray (nanoESI) source. NanoESI emitters with a tip ID of approximately $1 \mu \mathrm{m}$ were pulled from borosilicate capillaries with an ID of $0.78 \mathrm{~mm}$ using a Flaming-Brown P-97 micropipette puller (Sutter Instruments). For mass spectrometry analysis, $3-5 \mu \mathrm{L}$ of sample was loaded into an emitter, and electrospray was initiated by applying a potential (relative to instrument ground) of $+0.7-1.1 \mathrm{kV}$ to a platinum wire in electrical contact with the solution. All experiments were conducted in 
"Sensitivity" mode with the source equilibrated to ambient temperature and a backing pressure of 3.7 mbar. Traveling wave velocities of $400-550 \mathrm{~m} \mathrm{~s}^{-1}$ and wave heights of 18-25 V were used in all experiments. Nitrogen was used as the buffer gas in ion mobility experiments, and the helium cell and nitrogen gas

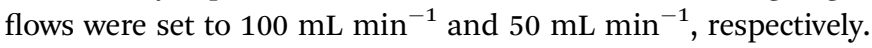
Measured arrival time distributions were converted to collisional cross sections using an established literature procedure. ${ }^{\mathbf{4 4 , 4 5}}$ Cytochrome C, $\beta$-lactoglobulin, avidin, bovine serum albumin, and concanavalin A were used as ion mobility calibrants.

\section{Collision-induced unfolding}

Collisional activation in the Waters Synapt G2-Si occurs in the "Trap" region of the instrument, located between the quadrupole and the ion mobility cell. A single charge state was isolated using the quadrupole with the LM Resolution set to 4.0 and the HM Resolution to 15. Collision-induced unfolding was performed by increasing the acceleration voltage (Trap CE) in $5 \mathrm{~V}$ increments, starting at $5 \mathrm{~V}$ and ending when significant fragmentation of the precursor ion was observed. The Trap gas flow was set to $5 \mathrm{~mL} \min ^{-1}$ for all collision-induced unfolding experiments. Arrival time distributions for each acceleration voltage were extracted using MassLynx 4.1 (Waters) and "fingerprint" images were generated using Igor Pro (WaveMetrics). For each acceleration voltage, the Multi Peak Fitting package in Igor Pro was used to fit the arrival time distribution with a number of Gaussian functions corresponding to the number of conformer families present. The area of each peak was computed to determine the relative abundance of each conformer family. For transitions resulting in at least 50\% conversion to the more unfolded conformer family in both SIU and CIU, appearance energies were determined by summing the relative abundances of each conformer family with a larger CCS than that of the initial family for the transition (i.e. for the transition out of conformer family 1 , sum the abundances of families 2, 3, and 4), and fitting that data with a sigmoidal function using Igor Pro - the appearance energy is the energy value at the intersection between the line tangent to the midpoint of the sigmoid and the $x$-axis.

\section{Surface-induced unfolding}

In the Synapt G2-Si, the SID device is located between the Trap and the ion mobility cell. ${ }^{25}$ Single charge state precursors were isolated in the manner described in the preceding section. Surface-induced unfolding was performed by increasing the SID acceleration potential in $5 \mathrm{~V}$ increments, starting at $20 \mathrm{~V}$ (the lowest SID voltage accessible) and continuing until fragmentation was observed. SID on this platform is accomplished by holding the surface at a fixed potential and then raising the potentials of all the instrument optics up to and including the exit electrode of the Trap by changing the "Trap DC Bias" value. Tuning of the entrance optics in the SID device is required to maintain ion transmission while causing the ions to hit the surface. The SID acceleration potential is calculated as the difference between the exit electrode of the Trap and the surface electrode. Minimally-activating conditions in the Trap were used: $5 \mathrm{~V}$ potential and $5 \mathrm{~mL} \mathrm{~min}^{-1}$ gas flow. Surface-induced unfolding data were analyzed using the procedure described for the collision-induced unfolding data.

\section{Combined unfolding: source + CIU or source + SIU}

On the Synapt G2-Si platform, activation prior to the quadrupole is readily accomplished by increasing the sampling cone voltage near the ion source. This value was set to either $130 \mathrm{~V}$ or $175 \mathrm{~V}$ for the experiments with BSA to produce mildly or moderately activated precursors, respectively, that were subsequently subjected to either collision- or surface-induced unfolding following the procedures outlined above. For the experiments combining in-source activation with SIU, the gas flow in the Trap was reduced to $1 \mathrm{~mL} \mathrm{~min}^{-1}$ to minimize any ion heating or cooling in that region.

\section{Monte Carlo and theoretical modeling of CIU}

For each protein and charge state studied experimentally, the CIU process was simulated at acceleration voltages from 10$200 \mathrm{~V}$ in $5 \mathrm{~V}$ increments using argon as the collision gas and two models of the activation process, one with a cooling mechanism and one without. A value of 0.9 was used for the per-collision efficiency of converting center-of-mass frame kinetic to internal energy, based on results for nonapeptides. ${ }^{46}$ Gas velocities were sampled from a Boltzmann distribution at a temperature of $298 \mathrm{~K}$, and collision geometries were sampled from an isotropic distribution. The collision probability at each time step was sampled from an exponential distribution consistent with the mean free path of the ion as derived from its CCS and the gas pressure in the Trap. The model for the on-axis electrical potential due to the traveling wave was adapted from Mortensen et al. ${ }^{47}$ To avoid undercounting collisions, the simulation time step was set to $1 / 20^{\text {th }}$ of the mean time between field free collisions at the highest initial kinetic energy for each protein simulated. Additionally, an analytic expression for the total internal energy deposited was derived in the limit of a large number of collisions and averaging over all possible collision geometries and gas velocities:

$$
\left\langle\Delta U^{\mathrm{tot}}\right\rangle=\frac{x N m_{\mathrm{i}}}{m_{\mathrm{i}}+m_{\mathrm{g}}}\left\langle\mathrm{KE}_{\mathrm{g}}^{\mathrm{lab}}\right\rangle+\frac{x\left(m_{\mathrm{i}}+m_{\mathrm{g}}\right)\left\langle\mathrm{KE}_{\mathrm{i}, 0}^{\mathrm{lab}}\right\rangle}{2 m_{\mathrm{i}}(1+\sqrt{1-x})+m_{\mathrm{g}} x}
$$

where $x$ is the fraction of available center-of-mass energy converted to internal energy, $N$ is the total number of collisions, $m_{\mathrm{i}}$ and $m_{\mathrm{g}}$ are the mass of the ion and gas, respectively, $\left\langle\mathrm{KE}_{\mathrm{g}}^{\mathrm{lab}}\right\rangle$ is the average kinetic energy of the gas, and $\left\langle\mathrm{KE}_{\mathrm{i}, 0}^{\mathrm{lab}}\right\rangle$ is the initial kinetic energy of the ion. Further details of the Monte Carlo simulations and derivation of eqn (1) can be found in the ESI. $\dagger$

\section{Results and discussion}

A major challenge in using SID (and CID) to determine quantitative energetic information is relating the nominal, laboratoryframe kinetic energy to the internal energy deposited into the ion. Because all of these experiments were performed on a Synapt $\mathrm{G} 2-\mathrm{Si}$ platform, the "laboratory-frame kinetic energy" ( $\left.\mathrm{KE}^{\mathrm{lab}}\right)$ is 
defined here as an ion's charge state multiplied by the voltage difference between either the FSAM-gold surface electrode and the exit electrode of the Trap in SID, or the entrance electrode of the Trap and exit electrode of the quadrupole in CID. The overall SIU or CIU efficiency is defined as the ratio of the initial $\mathrm{KE}^{\mathrm{lab}}$ and the final internal energy following the collision(s) or interaction with the surface. The relationship between initial $\mathrm{KE}^{\mathrm{lab}}$ and final internal energy may depend on factors such as the identity of the collision partner and number of collisions, and the mass, size, and shape of the ion.

\section{CIU and SIU access the same unfolding transitions}

Using the same instrumentation as for CID of protein complexes, accelerating a native-like protein monomer ion through a buffer gas often causes the ion to unfold and adopt one or more extended conformations at collision energies below those required for fragmentation. ${ }^{\mathbf{1 0}}$ The size and shape of these conformations can be assessed by ion mobility spectrometry prior to mass analysis. This technique, termed collisioninduced unfolding (CIU), has been demonstrated to yield useful information about a variety of protein structural characteristics. ${ }^{\mathbf{1 0 , 4 8 , 4 9}}$ Analogous protein unfolding using surface activation (surface-induced unfolding, SIU) has not yet been extensively studied. Comparing CIU and SIU for the same protein monomer ions is one approach to better understand the energy deposition (activation) process in SIU. Because CID typically partitions charge asymmetrically while SID partitions charge symmetrically, they often yield different products even for protein dimers. ${ }^{22,24}$ Since protein ions in CIU and SIU experiments do not significantly change mass or charge, their unfolding products and energies can be more directly compared than can dissociation products of protein oligomer ions.

As the first step in calibrating the internal energy deposited by SIU, 10 native-like protein ions, ranging in size from 17-80 $\mathrm{kDa}$, were unfolded using CIU and SIU. Results for three of these, bovine serum albumin $\left(\mathrm{BSA}^{15+}, 66 \mathrm{kDa}\right)$, the N-terminal domain of anthrax lethal factor $\left(\mathrm{LF}_{\mathrm{N}}{ }^{10+}, 31 \mathrm{kDa}\right)$, and transferrin $\left(\mathrm{TF}^{18+}, 80 \mathrm{kDa}\right)$ are illustrated in Fig. 1 as examples. For $\mathrm{BSA}^{15+}$, three structural transitions are observed in the CIU "fingerprint" between a native-like and three successively more unfolded conformer families as the nominal $\mathrm{KE}^{\mathrm{lab}}$ is increased from 300 to $2775 \mathrm{eV}$ (Fig. 1a). In the SIU "fingerprint" one native-like and three unfolded conformer families are observed with the same CCSs as in the CIU experiment, suggesting that the same unfolding transitions to the same unfolded states are taking place in both CIU and SIU of BSA (Fig. 1b). Similarly, $\mathrm{LF}_{\mathrm{N}}{ }^{10+}$ exhibits unfolding transitions from a native-like to three successively more unfolded conformer families in both CIU and SIU, with virtually identical CCSs in both data sets (Fig. 1c and d). In the case of TF, CIU causes a complete transition to the conformer family at approximately $58 \mathrm{~nm}^{2}$, and only minimal conversion to the conformer family at approximately $62 \mathrm{~nm}^{2}$ (Fig. 1e). However, SIU results in complete transitions to both unfolded conformer families (Fig. 1f), consistent with results from the Wysocki group showing that SID/SIU are able to effect greater activation on the timescale of the experiment than CID/
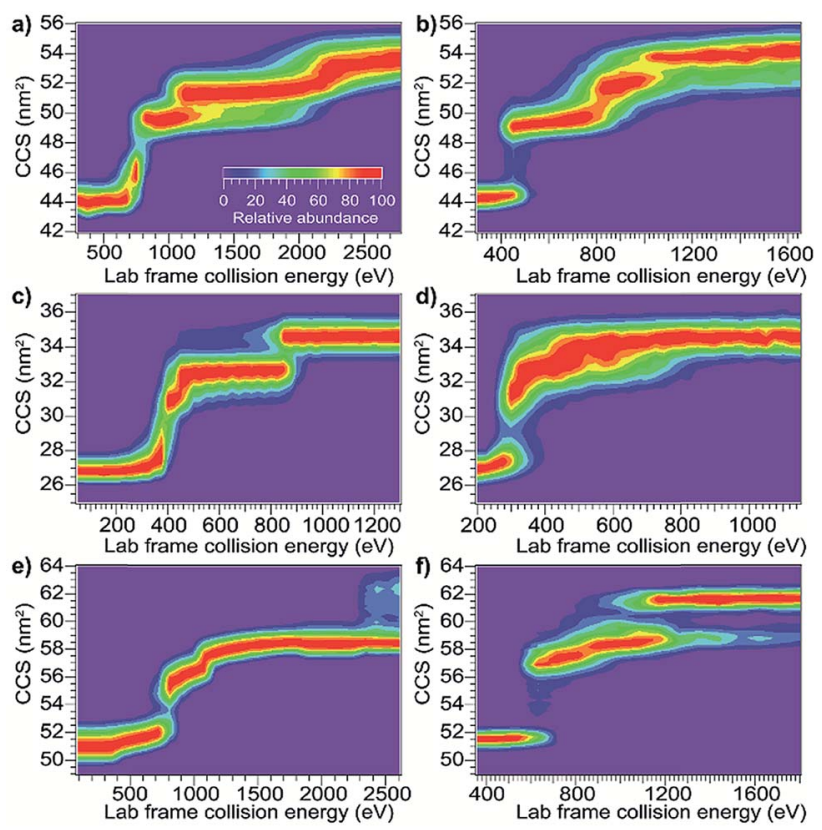

Fig. 1 Comparison of $\mathrm{CIU}$ and SIU for $\mathrm{BSA}^{15+}, \mathrm{LF}_{\mathrm{N}}{ }^{10+}$, and $\mathrm{TF}^{18+}$. (a) $\mathrm{CIU}$ of $\mathrm{BSA}^{15+}$ exhibiting three transitions to unfolded conformer families (b) SIU of $\mathrm{BSA}^{15+}$ with conformer families at the same CCSs as in $\mathrm{CIU}$ (c) $\mathrm{CIU}$ of $\mathrm{LF}_{\mathrm{N}}{ }^{10+}$ exhibiting three transitions to unfolded conformer families (d) SIU of $\mathrm{LF}_{\mathrm{N}}{ }^{10+}$ with conformer families at the same CCSs as in CIU (e) CIU of $\mathrm{TF}^{18+}$ exhibiting one full transition and the beginning of a second to unfolded conformer families ( $f$ ) SIU of $\mathrm{TF}^{18+}$ exhibiting two transitions to unfolded conformer families.

CIU at the same $\mathrm{KE}^{\mathrm{lab}}$. $^{7}$ Similar CIU and SIU analysis of the remainder of the protein monomers investigated shows that CIU and SIU produce unfolded states with the same CCSs for a variety of proteins and charge states (Fig. S1 $\dagger$ ).

Although CIU and SIU access the same conformer families, they do not produce identical unfolding "fingerprints" as a function of $\mathrm{KE}^{\mathrm{lab}}$. For each of the proteins studied, the CIU and SIU transitions occur at different acceleration voltages and thus different nominal laboratory frame kinetic energies. Additionally, the differences between the lab-frame appearance energies for successive structural transitions are not the same in CIU and SIU. However, defining the transition "appearance energy" as the energy at which the more unfolded conformer is detected (determined as described in the Methods section), extracting appearance energies for each transition, and plotting the nominal SIU energies against the nominal CIU energies reveals a correlation between SIU and CIU appearance energies (Fig. 2). The trend is clearly non-linear and can be empirically fit to a power law relationship with an exponent of $0.61 \pm 0.05$ (energy uncertainties are propagated from the uncertainty in the fit). Some of the scatter in the data set arises from uncertainty in the measurement of SIU or CIU energies (approximately $5 \%$, arising primarily from voltage fluctuations and uncertainty in the sigmoidal fits), but protein-specific factors, such as structure, almost certainly cause deviations from the fitted curve. However, neither the amount of $\alpha$-helical or $\beta$-sheet structure, the number of salt bridges, nor the ratios of CCS to 


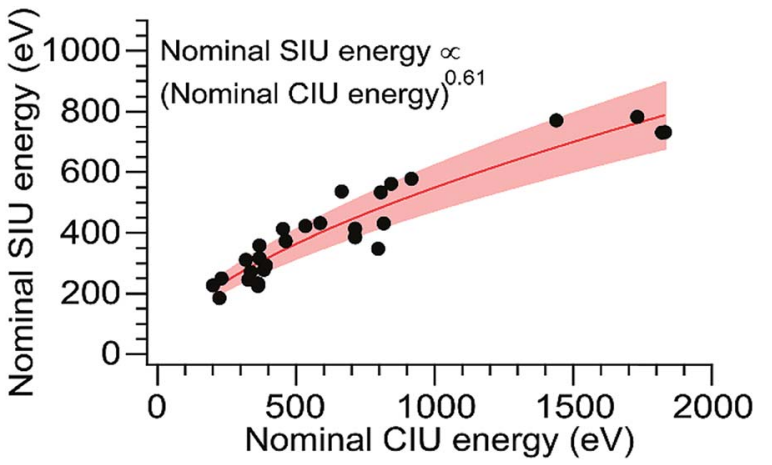

Fig. 2 Plot of SIU appearance energy vs. CIU appearance energy for a set of 10 protein monomers. The non-linear trend is fit to a power law relationship with an exponent of $0.61 \pm 0.05$. The shaded region represents \pm one standard deviation of the relative difference from the fit.

mass, charge to mass, and charge to CCS correlate with deviations from the fitted curve (Fig. S6 $\dagger$ ). The implication of the exponent being less than 1 is that SIU is relatively more efficient at causing unfolding than is $\mathrm{CIU}$ at higher $\mathrm{KE}^{\text {lab }}$ values under these instrumental conditions, due to an increase in SIU efficiency, a decrease in CIU efficiency, or some combination of both. Kinetic shifts may be present in both CIU and SIU on this instrument; however, the time for unfolding after activation is likely shorter in SIU because the SID device is located closer to the detector than is the Trap, where CIU occurs. This difference alone should result in a smaller kinetic shift for SIU compared to CIU. Furthermore, if kinetic shift alone were responsible for the non-linear trend observed in Fig. 2, the curvature of the trend should be in the opposite direction from what is observed. Thus, other factors beyond kinetic shift dominate the observed trend. The fit also intersects the origin, which implies that any other activation taking place that is not a function of the CIU or SIU potential is approximately the same for both processes under the conditions used here.

\section{Calibrating SIU energy deposition by modeling CIU energy deposition}

To relate the nominal SIU and CIU energies to the overall internal energy deposited into the ion by each process, the CIU process was modeled and used to calibrate the internal energy scale of the SIU data. The physics of the individual collisions involved in the CID/CIU process have been investigated previously, and a number of theoretical models describing the rate of ion heating and dissociation in multiple-collision CID have been introduced. ${ }^{50-53}$ In the Synapt G2-Si, in addition to the acceleration potential that injects ions into the collision cell there is a time-dependent electric field (traveling wave potential) that helps elute the ions out of the Trap. In order to model trajectories and energetics for protein ions undergoing CIU it is necessary to add this component to models describing the iongas collisions in a field-free environment, e.g., Douglas' model. ${ }^{\mathbf{1 1}}$ At the pressures used for CID/CIU in this instrument (approximately $2.6 \times 10^{-2} \mathrm{mbar}$ ), these protein ions experience from several hundred to several thousand collisions as they traverse the collision cell. Implementing a Monte Carlo simulation of the collision geometry and distance between collisions (a similar approach to that of Nesatyy and Laskin) ${ }^{50}$ enables consideration of both internal energy gain and kinetic energy loss due to energetic collisions.

For all proteins and charge states studied experimentally, the change in internal energy in both the presence and absence of cooling mechanisms is modeled for each $5 \mathrm{~V}$ increment of the acceleration potential from 10-200 V. The internal energy change increases linearly with $\mathrm{KE}^{\mathrm{lab}}$, and the slope of the trend line gives the overall CIU efficiency (Fig. 3). The overall CIU efficiency, defined as the maximum fraction of $\mathrm{KE}^{\mathrm{lab}}$ converted to internal energy, was found to be approximately $34 \%$ and decrease slightly with ion mass in the absence of a cooling mechanism, compared to $25 \%$ with a slight increase with ion mass with a cooling mechanism (Fig. S2 $\dagger$ ). These values provide upper and lower bounds to the energy deposition, since in our simulations collisional cooling occurs at the maximum possible rate. We expect that the actual amount of energy deposited is close to that computed in the simulations with cooling. When the collisions are highly inelastic (as in these experiments), the traveling wave has a small effect $(<2 \%)$ on the overall CIU efficiency (Fig. S3d $\dagger$ ), but does contribute to the non-zero intercepts of the plots in Fig. 3. Values of the total internal energy change and overall efficiency computed using eqn (1) are in excellent agreement with the results from Monte Carlo simulations without a cooling mechanism (Fig. S3a-c $\dagger$ ).
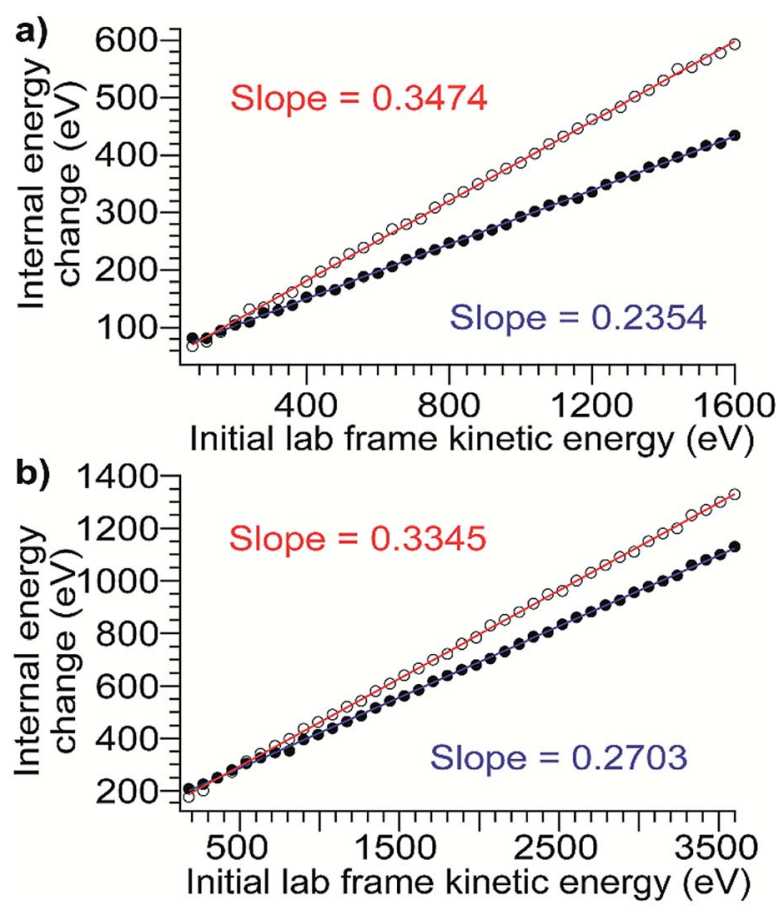

Fig. 3 Plots of internal energy change vs. initial laboratory-frame kinetic energy computed from Monte Carlo simulations using models with heating only (open circles, red line) and heating and cooling (filled circles, blue line) for (a) $\beta$-lactoglobulin ${ }^{8+}$ (b) $\mathrm{TF}^{18+}$. These plots are representative and in all cases there is a linear relationship. The slope of the trend line gives the overall CIU efficiency. 
Using the computed values for internal energy deposited during CIU (from the simulations with cooling), the energy axis for the data shown in Fig. 2 was calibrated (Fig. 4), with energy uncertainties in Fig. 4 propagated from the uncertainty in the fit. After this re-calibration, it is clear that energy deposition at higher laboratory frame collision energies is typically more efficient for SIU than for CIU, and SIU is more efficient for high-mass protein ions than for those with lower masses, reaching a maximum of $\sim 68 \%$ conversion to internal energy for the highest-energy transitions (using the CIU calibration with no cooling mechanism yields a value of $\sim 85 \%$, which we interpret as an extreme upper bound for energy deposition in SIU). Computational results for collisions of dialanine with an FSAM surface suggest that a maximum of $16 \%$ of initial $\mathrm{KE}^{\text {lab }}$ remains in translational modes after surface collision, ${ }^{35}$ leaving $84 \%$ or more of the energy to be partitioned between ion internal modes and the surface; an even lower fraction of the energy remains in translational modes for nanoscale polystyrene latex spheres after surface collision. ${ }^{54}$ Our maximum value falls within this upper bound, and suggests that energy transfer to internal modes dominates over energy transfer to the surface under these conditions. The nominal SIU energy vs. calibrated CIU energy trend can be fit to a power law relationship, with an exponent of $0.57 \pm 0.04$. The fact that the energy scale in SIU can be well-described by relation to CIU data implies that SIU, like CIU, can be understood as a primarily thermal process, in which the observed extent of unfolding in SIU is determined in large part by the amount of energy deposited into and equilibrated among the ion's internal modes. This result is in agreement with the majority of previous experimental and theoretical work on SID of small molecules, peptides, and proteins. ${ }^{30,31,55-57}$

Laskin and Futrell showed that in SID of peptides the efficiency of conversion to ion internal energy was approximately $20 \%$ and largely invariant to acceleration voltage. ${ }^{32,33}$ Thus, increased mass is likely to be primarily responsible for the observed increase in efficiency in our experiments. Previously published results for small molecules and peptides up to $1 \mathrm{kDa}$

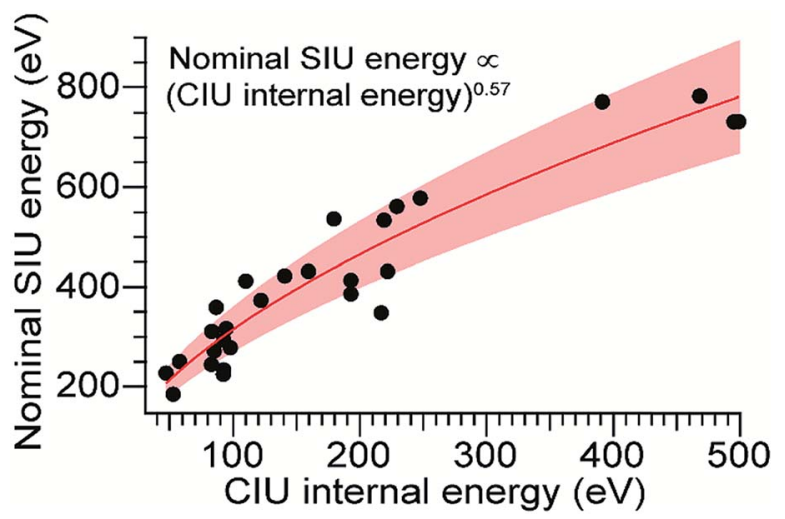

Fig. 4 SIU appearance energy vs. rescaled CIU internal energy. The non-linear trend is fit to a power law relationship with an exponent of $0.57 \pm 0.04$. The shaded region represents \pm one standard deviation of the relative difference from the fit. have shown that energy deposition efficiency varies little in this size range. ${ }^{32}$ The observed increase in overall SIU efficiency with mass in these experiments suggests that proteins of the size studied here fall into a different regime. Note that the SIU energy calibration presented here probes the overall deposition of energy into the ion and not dynamical details of how SIU energy deposition occurs, such as whether the ion interacts one or multiple times with the surface.

\section{In-source activation effects on SIU behavior}

Besides mass and initial kinetic energy, other factors that can in principle affect energy deposition in SID are the initial internal energy and structure of the protein ion. In-source ion activation followed by SIU was used to probe the influence of these factors. Applying a cone voltage of $130 \mathrm{~V}$ at a backing pressure of 3.7 mbar activates $\mathrm{BSA}^{15+}$ as much as possible without causing observable unfolding on the timescale of these experiments (see Fig. S8 $\uparrow$ for full in-source CIU fingerprint). Subjecting this activated precursor to SIU produces a remarkably similar unfolding "fingerprint" to that obtained without in-source activation (Fig. 5), and causes a $0.5 \%$ to $3.5 \%$ change in the unfolding transition appearance energies (Table 1). Thus, for a precursor with a given CCS, SIU under these conditions does not depend strongly on the degree of activation prior to the surface collision. This is in contrast to CIU, in which ions preactivated in the source region require less energy to unfold in the Trap (Fig. 6), with each transition shifted lower in energy. Interestingly, the size of the shift is the same for the first two unfolding transitions and half as large for the third unfolding transition (Table 1). These data indicate that in CIU ions retain a high internal temperature or already have many of their native non-covalent interactions disrupted as they enter the Trap. Decreased adduction following in-source activation may also contribute to the observed destabilization, as adducts have been shown to have a slight stabilizing effect in CIU, ${ }^{58-61}$ and in-
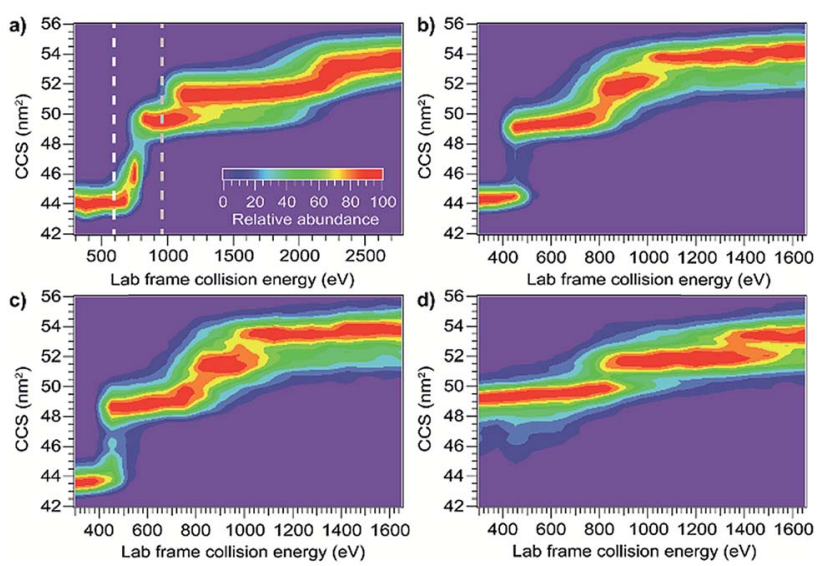

Fig. 5 Effect of in-source pre-activation on SIU of $\mathrm{BSA}^{15+}$. (a) CIU showing two different levels of in-source activation (b) SIU with no source activation (c) SIU following in-source activation to the white line in (a) does not differ from SIU with no source activation (d) SIU following in-source unfolding up to the gray line in (a) requires significantly higher energies to cause further unfolding transitions. 

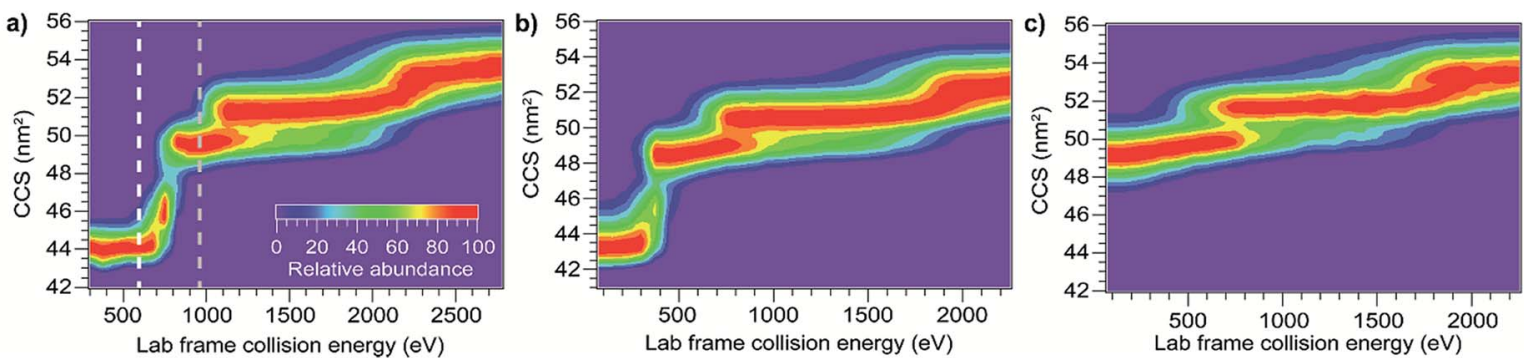

Fig. 6 Effect of pre-activation on CIU of $\mathrm{BSA}^{15+}$. (a) CIU showing two different levels of in-source activation (b) unfolding transitions in CIU following in-source activation up to the white line in (a) are shifted to lower energies (c) unfolding transitions in CIU following in-source unfolding up to the gray line in (a) are shifted to lower energies.

Table 1 Appearance energies for CIU and SIU with in-source activation

\begin{tabular}{|c|c|c|c|c|}
\hline \multirow{2}{*}{$\begin{array}{l}\text { Activation } \\
\text { method }\end{array}$} & \multirow{2}{*}{$\begin{array}{l}\text { Sampling cone } \\
\text { voltage }\end{array}$} & \multicolumn{3}{|c|}{ Appearance energies (eV) } \\
\hline & & 1 & 2 & 3 \\
\hline \multirow[t]{3}{*}{ CIU } & $25 \mathrm{~V}$ & 713 & 916 & 1731 \\
\hline & $130 \mathrm{~V}$ & 296 & 514 & 1518 \\
\hline & $175 \mathrm{~V}$ & & 288 & 1241 \\
\hline \multirow[t]{3}{*}{ SIU } & $25 \mathrm{~V}$ & 386 & 578 & 783 \\
\hline & $130 \mathrm{~V}$ & 377 & 598 & 779 \\
\hline & $175 \mathrm{~V}$ & & 649 & 953 \\
\hline
\end{tabular}

source activation in these experiments dissociates approximately 225 Da of adducts. However, for SIU, in-source activation has no significant effect on unfolding appearance energies, suggesting that these ions cool prior to SIU. Minimal cooling is observed for CIU with the same instrumental conditions between the source and the Trap, and SIU following preactivation in the Trap produces the same result as in-source pre-activation (Fig. S9†). Thus, cooling of the ions between the source and collision surface in SIU is likely due to collisions with helium or nitrogen leaking from the ion mobility cell.

Markedly different behavior is observed when the protein monomers studied here were measurably unfolded in the source region prior to SIU. For example, applying a cone voltage of $175 \mathrm{~V}$ with a backing pressure of 3.7 mbar causes $\mathrm{BSA}^{15+}$ to undergo an unfolding transition to the first unfolded conformer family (Fig. 5). The SIU fingerprint of this species is dramatically different from that generated without source unfolding (Fig. 5): the $\mathrm{KE}^{\mathrm{lab}}$ value required to precipitate the second unfolding transition increases by $71 \mathrm{eV}$, and the third unfolding transition is shifted higher in energy by $170 \mathrm{eV}$ (Table 1). Considering that the ions enter the Trap region partially unfolded, it is remarkable that more energy is required to precipitate the second and third unfolding transitions than without in-source activation, and cooling in the Trap cannot explain these differences. Thus, conversion of kinetic energy of the surface collision to internal energy of the protein must be much less efficient for these initially unfolded, relatively low-charge structures. The differences between the first and second and between the second and third transition appearance energies have increased by approximately $50 \%$, demonstrating that SIU is highly sensitive to the structure of the precursor ion; a 12\% increase in CCS leads to this dramatic reduction in the energy deposition efficiency. In simulations of triglycine SID, folded and extended structures differed little in energy deposition efficiency, ${ }^{34}$ again indicating that proteins of the size studied here fall into a different regime than peptides.

Contrasting behavior is observed in CIU, where in-source unfolding followed by CIU causes the second and third unfolding transition appearance energies to decrease by 628 and $490 \mathrm{eV}$, respectively (Table 1). These results agree with those for in-source activation without unfolding followed by CIU, and demonstrate that overall internal energy deposition efficiency for these ions in CIU depends on ion CCS to a much lesser extent than in SIU. This conclusion is supported by calculations of the overall CIU efficiency with cooling included, which show that although overall CIU efficiency depends non-linearly on CCS and has its maximum at the experimental CCS value of the native-like conformation (Fig. S4 $\dagger$ ), changing the CCS of $\mathrm{BSA}^{15+}$ by the amounts observed experimentally changes the overall CIU efficiency by only $1-5 \%$ (Fig. S5†). Simulations of collisional activation of peptides do show a small decrease in energy transfer for extended structures, a finding consistent with our results. ${ }^{62}$ SIU of pre-activated $\mathrm{TF}^{18+}$ provides a corroborating result, with in-source unfolding causing the appearance energy of the next transition to increase significantly (Fig. S10 $\dagger$ ).

These results suggest at least two mechanisms that contribute to the reduction in energy deposition efficiency for unfolded proteins in SIU (Fig. 7). First, the effective proteinsurface CCS can be smaller for unfolded proteins than for folded proteins, even though the protein-gas CCS is larger. That is, in many cases, an ion that is more elongated will on average interact with a smaller area of the surface than an ion with the same mass that is compact (Fig. 7). This reduces the effective center-of-mass frame kinetic energy of the collision and thus decreases the amount of energy transferred to internal modes. The increased flexibility of the unfolded protein ions in these experiments relative to the compact, native-like conformers likely also contributes to the lower energy conversion efficiency, because energy can be more efficiently transferred into rotational motion for more elongated structures. ${ }^{62}$ As rotation is less efficient than vibrational energy at causing further unfolding, ${ }^{63}$ 

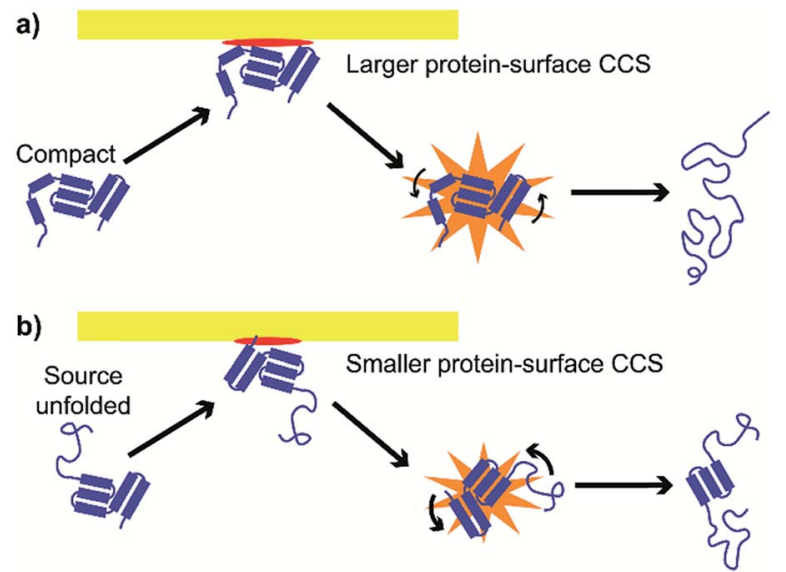

Fig. 7 Effective protein-surface CCS is larger and transfer to rotational modes less efficient for (a) compact structures than for (b) unfolded structures, causing energy deposition efficiency to be higher for compact structures.

this will increase the amount of $\mathrm{KE}^{\mathrm{lab}}$ required to cause further unfolding. Multiple interactions of the ion with the surface may further complicate energy deposition.

\section{Conclusion}

The energy deposition process in SID of proteins was studied by comparing unfolding of protein monomers by collisional and surface activation and by investigating the effect of in-source pre-activation on CIU and SIU. CIU and SIU cause similar unfolding transitions and the conformer families observed have the same CCS. The nominal energy scales of CIU and SIU are correlated and follow a power law relationship, with SIU being more efficient than CIU at higher $\mathrm{KE}^{\text {lab }}$ values and for larger ions, but significant scatter from this trend indicates other protein-specific factors also play a significant role, although no correlations in deviations from the trend were found with respect to secondary structure content, number of salt bridges, or the ratios of charge to mass, charge to CCS, or CCS to mass. These factors may be structural or may be related to the mechanical protein-surface interaction. The average overall efficiency for conversion of initial $\mathrm{KE}^{\mathrm{lab}}$ to internal energy for CIU determined by Monte Carlo simulations including both ion heating and cooling is approximately $25 \%$, increasing slightly with ion mass. These calculated data are used to calibrate the SIU energy scale, leading to computed efficiency values for SIU of up to $68 \%$ for the largest ions studied $(80 \mathrm{kDa})$. Alternatively, this is equivalent to the effective mass of the surface during the collision, defined as the mass of the effective two-body collision partner required to impart a center-of-mass frame energy equal to the calibrated CIU internal energy, ${ }^{55}$ increasing with protein size. Previous determinations of the effective mass of the surface in peptide SID experiments were a few tens of Da, ${ }^{55,56}$ a result corroborated by atomistic simulations of peptide SID showing that the peptide interacts with a single $-\mathrm{CF}_{3}$ group (mass of $69 \mathrm{Da}$ ). ${ }^{35}$ Our data suggest that the effective mass of the surface grows with the mass of the protein (Fig. S11†).
When in-source pre-activation is followed by CIU, for both degrees of in-source activation (unfolded and not unfolded), subsequent unfolding transitions are shifted to lower energies by an amount commensurate with the amount of in-source activation. This indicates that effects of activation, i.e. an elevated internal temperature or disruption of non-covalent interactions, can be preserved as the protein ions travel from the source region to the entrance of the Trap. In contrast, preactivation without unfolding has little effect on SIU, indicating that protein ions are cooled between the end of the Trap and the SID device. Protein ions unfolded in the source require significantly more energy to further unfold in SIU than their compact counterparts, showing that energy deposition in SID is sensitive to ion CCS, with reduced conversion efficiency for more unfolded ions due to a smaller effective protein-surface CCS and increased transfer to rotational modes. The Wysocki group has combined in-source activation with SID of protein tetramers and demonstrated that when partially-unfolded complexes are subjected to SID they produce fragments akin to those produced by CID. ${ }^{64}$ The results obtained here parallel those and provide quantitative insights into the effect of structure on the SID energy deposition process. Future experiments probing the energy required to dissociate pre-activated protein complexes could elucidate how the effects demonstrated here change with varying complex size and number of subunits. We anticipate that calibration of energy deposition in SID using a strategy similar to that reported here may enable more quantitative calibration of SID appearance energies with respect to the disrupted protein-protein interfacial areas, facilitating rapid characterization of protein-protein interactions in previously unexamined complexes, including membrane protein complexes. Future investigation of the role of protein structure as well as mechanical effects of the surface collision, including those revealed by sophisticated chemical dynamics simulations ${ }^{65}$ are expected to shed light on proteinspecific deviations from the SIU-CIU calibration curve described here.

\section{Conflicts of interest}

There are no conflicts of interest to declare.

\section{Acknowledgements}

The authors thank Jeff Cina for helpful conversations. M. T. D. acknowledges support from a National Science Foundation Graduate Research Fellowship (DGE-1309047). Research reported in this publication was supported by the National Institutes of Health under award number R21AI125804 (to J. S. P.). The content is solely the responsibility of the authors and does not necessarily represent the official views of the National Science Foundation or the National Institutes of Health.

\section{References}

1 F. Lanucara, S. W. Holman, C. J. Gray and C. E. Eyers, Nat. Chem., 2014, 6, 281-294. 
2 A. Resemann, D. Wunderlich, U. Rothbauer, B. Warscheid, H. Leonhardt, J. Fuchser, K. Kuhlmann and D. Suckau, Anal. Chem., 2010, 82, 3283-3292.

3 N. Siuti, M. J. Roth, C. A. Mizzen, N. L. Kelleher and J. J. Pesavento, J. Proteome Res., 2006, 5, 233-239.

4 J. B. Shaw, W. Li, D. D. Holden, Y. Zhang, J. Griep-Raming, R. T. Fellers, B. P. Early, P. M. Thomas, N. L. Kelleher and J. S. Brodbelt, J. Am. Chem. Soc., 2013, 135, 12646-12651.

5 J. N. Rabuck-Gibbons, J. E. Keating and B. T. Ruotolo, Int. J. Mass Spectrom., 2018, 427, 151-156.

6 X. Ma, J. A. Loo and V. H. Wysocki, Int. J. Mass Spectrom., 2015, 377, 201-204.

7 X. Ma, M. Zhou and V. H. Wysocki, J. Am. Soc. Mass Spectrom., 2014, 25, 368-379.

8 Y. Song, M. T. Nelp, V. Bandarian and V. H. Wysocki, ACS Cent. Sci., 2015, 1, 477-487.

9 J. L. P. Benesch, J. A. Aquilina, B. T. Ruotolo, F. Sobott and C. V. Robinson, Chem. Biol., 2006, 13, 597-605.

10 S. M. Dixit, D. A. Polasky and B. T. Ruotolo, Curr. Opin. Chem. Biol., 2018, 42, 93-100.

11 R. A. Zubarev, Curr. Opin. Biotechnol., 2004, 15, 12-16.

12 R. A. Zubarev, N. L. Kelleher and F. W. McLafferty, J. Am. Chem. Soc., 1998, 120, 3265-3266.

13 J. J. Coon, J. Shabanowitz, D. F. Hunt and J. E. P. Syka, J. Am. Soc. Mass Spectrom., 2005, 16, 880-882.

14 J. E. P. Syka, J. J. Coon, M. J. Schroeder, J. Shabanowitz and D. F. Hunt, Proc. Natl. Acad. Sci. U. S. A., 2004, 101, 95289533.

15 S. A. McLuckey, J. Am. Soc. Mass Spectrom., 1992, 3, 599-614. 16 J. S. Brodbelt, Chem. Soc. Rev., 2014, 43, 2757-2783.

17 J. P. Reilly, Mass Spectrom. Rev., 2009, 28, 425-447.

18 J. S. Brodbelt and J. J. Wilson, Mass Spectrom. Rev., 2009, 28, 390-424.

19 B. L. Schwartz, J. E. Bruce, G. A. Anderson, S. A. Hofstadler, A. L. Rockwood, R. D. Smith, A. Chilkoti and P. S. Stayton, J. Am. Soc. Mass Spectrom., 1995, 6, 459-465.

20 J. C. Jurchen and E. R. Williams, J. Am. Chem. Soc., 2003, 125, 2817-2826.

21 S. V. Sciuto, J. Liu and L. Konermann, J. Am. Soc. Mass Spectrom., 2011, 22, 1679.

22 R. L. Beardsley, C. M. Jones, A. S. Galhena and V. H. Wysocki, Anal. Chem., 2009, 81, 1347-1356.

23 M. Zhou, S. Dagan and V. H. Wysocki, Analyst, 2013, 138, 1353-1362.

24 C. M. Jones, R. L. Beardsley, A. S. Galhena, S. Dagan, G. Cheng and V. H. Wysocki, J. Am. Chem. Soc., 2006, 128, 15044-15045.

25 M. Zhou, S. Dagan and V. H. Wysocki, Angew. Chem., Int. Ed., 2012, 51, 4336-4339.

26 R. S. Quintyn, J. Yan and V. H. Wysocki, Chem. Biol., 2015, 22, 583-592.

27 M. Zhou, C. M. Jones and V. H. Wysocki, Anal. Chem., 2013, 85, 8262-8267.

28 J. Yan, M. Zhou, J. D. Gilbert, J. J. Wolff, Á. Somogyi, R. E. Pedder, R. S. Quintyn, L. J. Morrison, M. L. Easterling, L. Paša-Tolić and V. H. Wysocki, Anal. Chem., 2017, 89, 895-901.
29 J. Laskin, E. V. Denisov, A. K. Shukla, S. E. Barlow and J. H. Futrell, Anal. Chem., 2002, 74, 3255-3261.

30 A. S. Galhena, S. Dagan, C. M. Jones, R. L. Beardsley and V. H. Wysocki, Anal. Chem., 2008, 80, 1425-1436.

31 J. Žabka, Z. Dolejšek and Z. Herman, J. Phys. Chem. A, 2002, 106, 10861-10869.

32 J. Laskin and J. H. Futrell, J. Chem. Phys., 2002, 116, 43024310.

33 J. Laskin and J. H. Futrell, J. Am. Soc. Mass Spectrom., 2003, 14, 1340-1347.

34 O. Meroueh and W. L. Hase, J. Am. Chem. Soc., 2002, 124, 1524-1531.

35 S. Pratihar, S. C. Kohale, D. G. Bhakta, J. Laskin and W. L. Hase, Phys. Chem. Chem. Phys., 2014, 16, 23769-23778.

36 T. Raz and R. D. Levine, Chem. Phys., 1996, 213, 263-275.

37 J. Laskin, T. H. Bailey and J. H. Futrell, J. Am. Chem. Soc., 2003, 125, 1625-1632.

38 S. O. Meroueh, Y. Wang and W. L. Hase, J. Phys. Chem. A, 2002, 106, 9983-9992.

39 K. Park, B. Deb, K. Song and W. L. Hase, J. Am. Soc. Mass Spectrom., 2009, 20, 939-948.

40 E. Hendell, U. Even, T. Raz and R. D. Levine, Phys. Rev. Lett., 1995, 75, 2670-2673.

41 T. Raz and R. D. Levine, J. Chem. Phys., 1996, 105, 8097-8102. 42 R. D. Beck, C. Warth, K. May and M. M. Kappes, Chem. Phys. Lett., 1996, 257, 557-562.

43 D. G. Schultz and L. Hanley, J. Chem. Phys., 1998, 109, 1097610983.

44 M. F. Bush, Z. Hall, K. Giles, J. Hoyes, C. V. Robinson and B. T. Ruotolo, Anal. Chem., 2010, 82, 9557-9565.

45 B. T. Ruotolo, J. L. P. Benesch, A. M. Sandercock, S.-J. Hyung and C. V. Robinson, Nat. Protoc., 2008, 3, 1139-1152.

46 E. M. Marzluff, S. Campbell, M. T. Rodgers and J. L. Beauchamp, J. Am. Chem. Soc., 1994, 116, 6947-6948.

47 D. N. Mortensen, A. C. Susa and E. R. Williams, J. Am. Soc. Mass Spectrom., 2017, 28, 1282-1292.

48 Y. Tian, L. Han, A. C. Buckner and B. T. Ruotolo, Anal. Chem., 2015, 87, 11509-11515.

49 Y. Zhong, L. Han and B. T. Ruotolo, Angew. Chem., Int. Ed., 2014, 53, 9209-9212.

50 V. J. Nesatyy and J. Laskin, Int. J. Mass Spectrom., 2002, 221, 245-262.

51 D. J. Douglas, J. Am. Soc. Mass Spectrom., 1998, 9, 101-113.

52 A. Hoxha, C. Collette, E. De Pauw and B. Leyh, J. Phys. Chem. A, 2001, 105, 7326-7333.

53 E. Uggerud and P. J. Derrick, J. Phys. Chem., 1991, 95, 14301436.

54 B. D. Adamson, M. E. C. Miller and R. E. Continetti, EPJ Techniques and Instrumentation, 2017, 4, 2.

55 J. Laskin, E. Denisov and J. Futrell, J. Am. Chem. Soc., 2000, 122, 9703-9714.

56 J. Laskin, E. Denisov and J. Futrell, J. Phys. Chem. B, 2001, 105, 1895-1900.

57 V. S. Rakov, E. V. Denisov, J. Laskin and J. H. Futrell, J. Phys. Chem. A, 2002, 106, 2781-2788.

58 L. Han, S.-J. Hyung, J. J. S. Mayers and B. T. Ruotolo, J. Am. Chem. Soc., 2011, 133, 11358-11367. 
59 L. Han, S.-J. Hyung and B. T. Ruotolo, Angew. Chem., Int. Ed., 2012, 51, 5692-5695.

60 J. Freeke, C. V. Robinson and B. T. Ruotolo, Int. J. Mass Spectrom., 2010, 298, 91-98.

61 N. D. Wagner, D. Kim and D. H. Russell, Anal. Chem., 2016, 88, 5934-5940.

62 O. Meroueh and W. L. Hase, Int. J. Mass Spectrom., 2000, 201, 233-244.
63 T. Baer, W. L. Hase, W. L. Hase and W. L. Hase, Unimolecular Reaction Dynamics: Theory and Experiments, Oxford University Press, USA, 1996.

64 R. S. Quintyn, M. Zhou, J. Yan and V. H. Wysocki, Anal. Chem., 2015, 87, 11879-11886.

65 S. Pratihar, S. C. Kohale, S. A. Vázquez and W. L. Hase, J. Phys. Chem. B, 2014, 118, 5577-5588. 